\title{
Multi-Stimuli-Triggered Release of Charged Dye from Smart PEGylated Nanogels Containing Gold Nanoparticles to Regulate Fluorescence Signals $\uparrow$
}

\author{
Motoi Oishi $^{a, b, c}$, Takahito Nakamura ${ }^{a}$, Yuta Jinji ${ }^{a}$, Kiyoto Matsuishi ${ }^{a}$, and Yukio Nagasaki ${ }^{*}{ }^{* a b, c, d, e}$ \\ ${ }_{5}$ Received (in $\left.X X X, X X X\right)$ Xth $X X X X X X X X X 200 X$, Accepted Xth $X X X X X X X X X 200 X$ \\ First published on the web Xth $X X X X X X X X X 200 X$ \\ DOI: 10.1039/b000000x
}

Polyion complex nanoparticles based on 1,3,6,8pyrenetetrasulfonic acid tetrasodium salt (PSA) and stimuli10 responsive PEGylated nanogels composed of PEG tethered chains and a cross-linked poly[2-(N,N-diethylamino)ethyl methacrylate] gel core containing gold nanoparticles showed a remarkable regulation of the fluorescence signal synchronizing with the multi-stimuli-triggered release of the PSA in response to 15 pH, temperature and light.

Polyion complex (PIC) nanoparticles have attracted much attention in the field of biological and biomedical applications, because they are readily embedded with charged hydrophilic molecules such as protein, ${ }^{1,2}$ DNA, ${ }^{3,4}$ probe molecules, ${ }^{5,6}$ and 20 drugs $^{7,8}$ through electrostatic interactions. Recent interest has surrounded a report on the development of stimuli-responsive PIC nanoparticles that can be triggered to release the charged hydrophilic molecules in response to stimuli, such as $\mathrm{pH},{ }^{9,10}$ redox compounds inside the cell, ${ }^{11-13}$ ions, ${ }^{14,15}$ electric fields, ${ }^{16}$ 25 temperature, ${ }^{17,18}$ enzymes, ${ }^{19,20}$ light, ${ }^{21,22}$ and so on. Nevertheless, most of the stimuli-responsive PIC nanoparticles mentioned above are able to release the charged hydrophilic molecules only in response to single stimuli. Accordingly, the development of multi-stimuli-responsive ("smart") PIC nanoparticles is of great 30 significance in both fundamental and applied scientific research. Worth noticing in this regard is that we have recently reported smart PEGylated nanogels constructed from a cross-linked core of poly[2-( $N, N$-diethylamino)ethyl methacrylate] (PEAMA) gel and tethered PEG chains that possess a carboxylic acid or an 35 acetal group as a platform for the installation of ligand molecules. ${ }^{23,24}$ The PEGylated nanogels showed extremely high dispersion stability as well as reversible volume-phase transition (swelling-deswelling) in response to multi-stimuli such as $\mathrm{pH}^{23,24}$ temperature, $^{23}$ and ionic strength ${ }^{23}$ due to the protonation40 deprotonation of the cross-linked PEAMA core surrounded by the tethered PEG chains. Indeed, the PEGylated nanogels can be utilized as $\mathrm{pH}$-triggered release carriers for drug delivery systems ${ }^{25}$ as well as $\mathrm{pH}$-responsive nano-probes for ${ }^{19} \mathrm{~F}$ MRI, ${ }^{26,27}$ and, importantly, the PEGylated nanogels form PIC nanoparticles 45 with negatively charged siRNA. ${ }^{28}$ Worth noticing is that the cross-linked PEAMA core of the PEGylated nanogels was found to act as a nano-reactor and nano-matrix to produce and immobilize the gold nanoparticles (GNPs) through the reduction of chloroaurate ions without any additional reducing agents. ${ }^{29,30}$ ${ }_{50}$ GNPs are known to absorb light at their unique and sensitive surface plasmon band (SPB) and generate heat efficiently through the photothermal effect within the vicinity of the GNP. ${ }^{31-33}$

Herein, we describe our study of the polyion complexation between highly water-soluble pyrene as a negatively charged 55 fluorescent probe and PEGylated nanogels containing GNPs (PEGylated GNGs) in aqueous media. A unique finding, which we would like to communicate here, is the $\mathrm{pH}-$, thermo-, and photothermally-triggered release of 1,3,6,8-pyrenetetrasulfonic acid tetrasodium salt (PSA) from PEGylated GNGs, which 60 allowed the regulation of the fluorescence signal from the PSA. In particular, the combination of PEGylated GNGs and light as an external stimulus may represent a promising strategy for the spatially and temporally controlled release of the probe molecules (Fig. 1).

65 PEGylated nanogel was synthesized by the emulsion copolymerization of 2-(N,N-diethylamino)ethyl methacrylate (EAMA) and heterobifunctional PEG bearing an acetal group at the $\alpha$-end and a 4-vinylbenzyl group at the $\omega$-end (acetal-PEG-
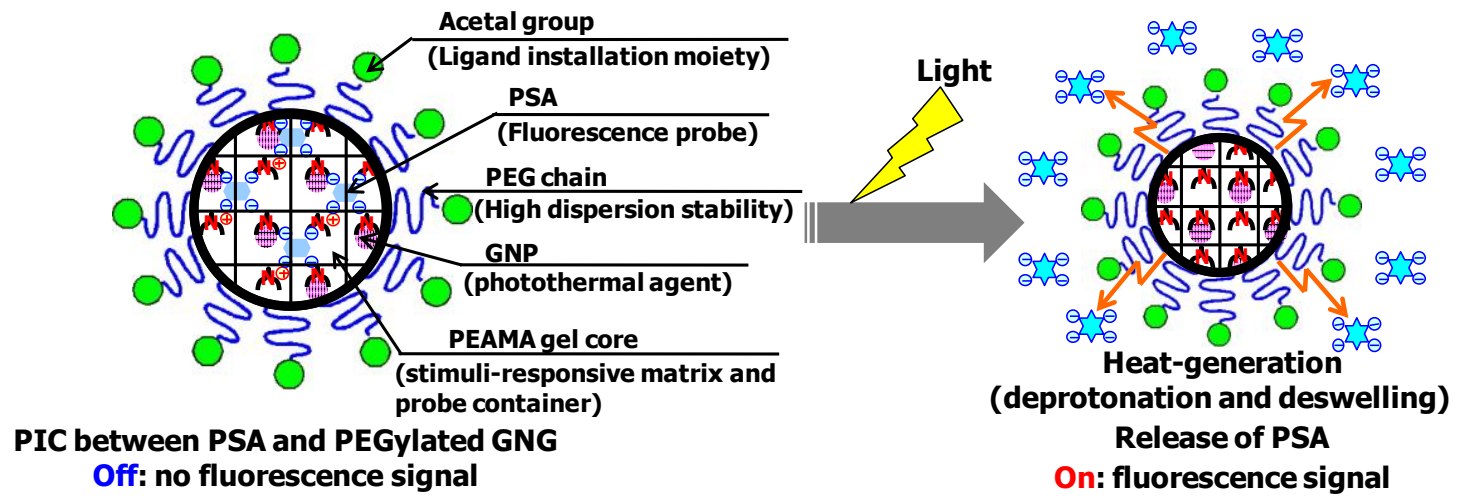

Figure 1. Schematic illustration of a PIC nanoparticle prepared from PSA and PEGylated GNG and the photothermally-triggered release of PSA from the PEGylated GNG. 
a)

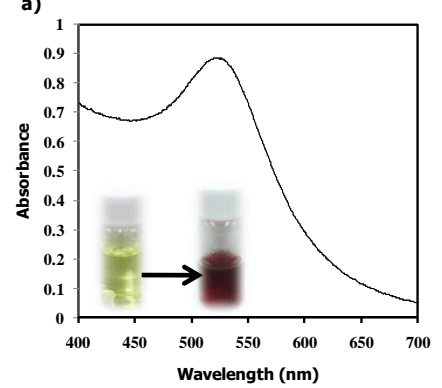

Figure 2. a) UV-vis spectrum, and b) TEM image of the PEGylated GNG prepared at $\mathrm{pH} 6$.

Ph-CH= $\left.\mathrm{CH}_{2}: M_{\mathrm{n}}=7,200, M_{\mathrm{w}} / M_{\mathrm{n}}=1.03\right)$, and $1.0 \mathrm{~mol} \%$ of ethylene glycol dimethacrylate (cross-linker) in the presence of potassium persulfate (initiator). The diameter of the PEGylated nanogel increased with decreasing temperature from 50 (diameter $\left.{ }_{5}=93 \mathrm{~nm}\right)$ to $15^{\circ} \mathrm{C}($ diameter $=187 \mathrm{~nm})$ at $\mathrm{pH} 6$ (see Fig. $\mathrm{S} 1$ in ESI). The thermo-induced size variation of the PEGylated nanogel at $\mathrm{pH} 6$ was due to the increase in the ion osmotic pressure as well as the solvation of the PEAMA gel core caused by the change in the degree of protonation $(\alpha)$ of the tertiary 10 amino groups at $\mathrm{pH} 6$ in response to the temperature $\left(15^{\circ} \mathrm{C}: \alpha=\right.$ $0.6,25^{\circ} \mathrm{C}: \alpha=0.5,50^{\circ} \mathrm{C}: \alpha=0.1$, see Fig. S2 in ESI).

In accordance with our previous report, ${ }^{30}$ the preparation of PEGylated GNGs was carried out at N/Au=2 (molar ratio of the amino groups in the PEGylated nanogel to $\mathrm{HAuCl}_{4}$ ) by the 15 reduction of tetrachloroaurate acid $\left(\mathrm{HAuCl}_{4}\right.$ (III)) with PEGylated nanogel at $\mathrm{pH} 6.0$ without any additional reducing agents. In this reaction, the tertiary amino groups in the PEAMA gel core act as reducing agents (nano-reactor) as well as stabilizers of the GNPs through the coordination between the 20 gold surface and the amino groups (nano-matrix). After the reduction of $\mathrm{HAuCl}_{4}$, an increase in the absorbance at around 520 nm (i.e., SPB) was also observed (Fig. 2a), and the color of the PEGylated nanogel solution changed to red (Fig. 2a). The transmission electron microscopy (TEM) image of the PEGylated ${ }_{25}$ GNGs shows that GNP clusters were observed, where the TEM shows not only GNPs (high contrast) but also the PEAMA gel core of the nanogel (lower contrast; $<100 \mathrm{~nm}$ ), as can be seen in Fig. 2b. These facts strongly suggest that the formation of GNPs in the PEAMA gel core occurred. The average number of GNPs 30 in a single PEAMA gel core and the average diameter of the GNPs were about $15.8 \pm 6.8$ and $7.7 \pm 2.7 \mathrm{~nm}$, respectively.

Fig. 3 shows the temperature increments $(\Delta \mathrm{T})$ for PEGylated GNG $(81 \mu \mathrm{g} / \mathrm{mL},[\mathrm{Au}]=24 \mu \mathrm{g} / \mathrm{mL})$ aqueous solution under irradiation with a $600 \mathrm{~mW}$ Ar ion $\left(\mathrm{Ar}^{+}\right)$laser $(\lambda=514.5 \mathrm{~nm})$ at a 35 fluence of $39 \mathrm{~W} / \mathrm{cm}^{2}$ for $10 \mathrm{~min}\left(23.4 \mathrm{~kJ} / \mathrm{cm}^{2}\right)$. Note, that the solution temperature gradually increased with the increase in the laser irradiation time, and, consequently, the solution temperature changed from $25^{\circ} \mathrm{C}$ to $32.3^{\circ} \mathrm{C}\left(\Delta \mathrm{T}=8.1^{\circ} \mathrm{C}\right)$. In sharp contrast, the PEGylated nanogel aqueous solution $(57 \mu \mathrm{g} / \mathrm{mL},[\mathrm{Au}]=0$ $40 \mu \mathrm{g} / \mathrm{mL}$ ) showed a negligible increment of the solution temperature even under laser-irradiation $\left(\Delta \mathrm{T}<1.0^{\circ} \mathrm{C}\right)$. These facts strongly indicate that the increment of the solution temperature under laser irradiation was due to the efficient generation of heat from the GNPs in the PEGylated GNGs 45 (photothermal effect).

To form PIC nanoparticles from PEGylated GNG and

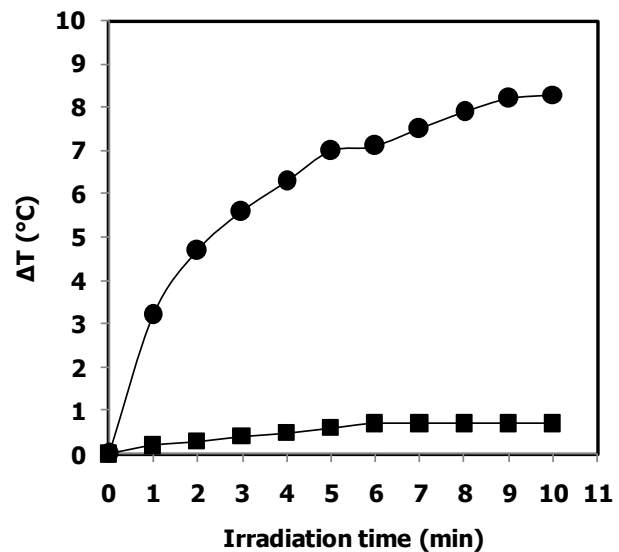

Figure 3. Temperature increments $(\Delta \mathrm{T})$ of the PEGylated GNG solution (circle) and PEGylated nanogel solution (square) as a function of the irradiation time with a $600 \mathrm{~mW} \mathrm{Ar}^{+}$laser at a fluence of $39 \mathrm{~W} / \mathrm{cm}^{2}\left(\lambda=514.5 \mathrm{~nm}, 23.4 \mathrm{~kJ} / \mathrm{cm}^{2}\right)$.

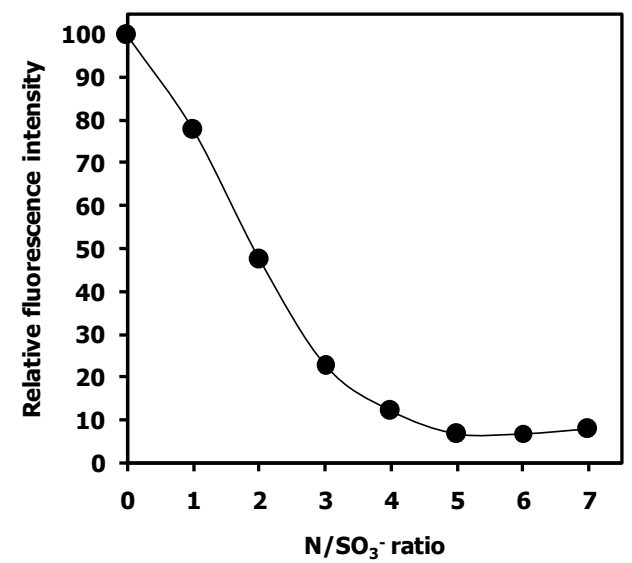

Figure 4. Relative fluorescence intensity of the PSA in the presence of the PEGylated GNG at $\mathrm{pH} 3.5$ for various $\mathrm{N} / \mathrm{SO}_{3}{ }^{-}$ ratios.

negatively charged PSA, various amounts of PEGylated GNG solution were added to a PSA solution, and all the mixtures were adjusted to $\mathrm{pH} 3.5$ and a PSA concentration of $10 \mu \mathrm{M}$. At this ${ }_{50} \mathrm{pH}$, the amino groups of the PEGylated GNG were completely protonated, as determined from its $\alpha / \mathrm{pH}$ curve (see Fig. S3 in ESI). Fig. 4 shows the relative fluorescence intensity of the PSA in the presence of various amounts of PEGylated GNGs as a function of theN/SO${ }_{3}^{-}$ratio (amino groups in the PEGylated ${ }_{55} \mathrm{GNG} /$ sulfonate groups in PSA). Note, that the free PSA (at $\mathrm{N} / \mathrm{SO}_{3}{ }^{-}=0$ ) showed fluorescence with maxima at 386 and 406 $\mathrm{nm}$. In sharp contrast, the relative fluorescence intensity of PSA in the presence of the PEGylated GNG decreased significantly with an increase in the $\mathrm{N} / \mathrm{SO}_{3}{ }^{-}$ratio and reached a plateau value 60 of around $7 \%$ at $\mathrm{N} / \mathrm{SO}_{3}{ }^{-}$ratios higher than 5 (i.e., off-state). These facts indicate that the change in fluorescence intensity was due to the aggregation of the PSA in the PEAMA gel core (selfquenching effect) caused by the formation of a PIC between the PSA and the PEAMA gel core; viz., the complete encapsulation ${ }_{65}$ of the PSA (polyion complexation) was attained in the $\mathrm{N} / \mathrm{SO}_{3}{ }^{-}$ range of $>5$. Worth noticing is that the fluorescence intensity of the PSA in the presence of PEGylated GNGs at $\mathrm{N} / \mathrm{SO}_{3}{ }^{-}=5$ almost recovered, when the $\mathrm{pH}$ of the solution was changed from 3.5 to 9.5 to cause the deprotonation of the PEAMA gel core $(\mathrm{pH}$ 


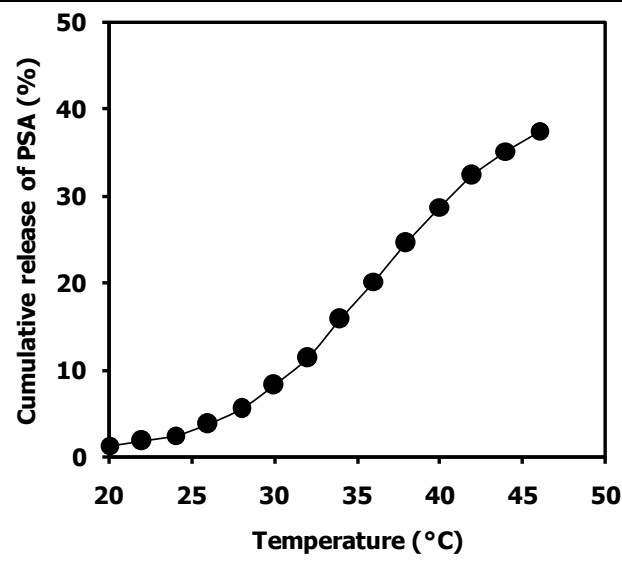

Figure 5. Release profile of the PSA from the PSA-loaded PEGylated GNG at $\mathrm{pH} 8$ as a function of temperature. The fluorescence spectra were measured after allowing the PSAloaded PEGylated GNG solution to stand in the cell at a given temperature.

3.5: $\alpha=1.0$ and $\mathrm{pH}$ 9.5: $\alpha=0$ ), suggesting the $\mathrm{pH}$-triggered release of the PSA from the PEGylated GNG (pH 3.5: off-state and $\mathrm{pH}$ 9.5: on-state) (see Fig. S4 in ESI).

The release of the PSA from the PSA-loaded PEGylated GNGs 5 prepared at $\mathrm{N} / \mathrm{SO}_{3}{ }^{-}=6$ was carried out in $10 \mathrm{mM}$ phosphate buffer (PB) at $\mathrm{pH} 8.0$ as a function of temperature, and the release profile is shown in Fig.5. As the temperature increased from $20^{\circ} \mathrm{C}$ (off-state), the fluorescence intensity increased gradually, and, eventually, approximately $40 \%$ of the PSA was released 10 from the PSA-loaded PEGylated GNGs at $46^{\circ} \mathrm{C}$ (on-state). This is due to the volume-phase transition of the PEAMA gel core in response to the temperature; viz., the thermo-triggered release of the PSA occurred due to the disappearance of the positive charges through the deprotonation of the PEAMA gel core. Unfortunately, 15 the release profile of the PSA almost reached a plateau, viz., approximately $60 \%$ of the PSA still remained in the PEGylated GNGs even at $46^{\circ} \mathrm{C}$. Further studies on the effect of the incubation time and higher temperature are probably required to achieve the complete release of the PSA from the PEGylated ${ }_{20}$ GNG.

The release of the PSA from PSA-loaded PEGylated GNGs prepared at $\mathrm{N} / \mathrm{SO}_{3}{ }^{-}=6$ was carried out in $10 \mathrm{mM} \mathrm{PB}$ containing $0.15 \mathrm{M} \mathrm{NaCl}$ at $\mathrm{pH} 7.4$ under irradiation with a $600 \mathrm{~mW} \mathrm{Ar}^{+}$ laser $(\lambda=514.5 \mathrm{~nm})$ at a fluence of $39 \mathrm{~W} / \mathrm{cm}^{2}$ as a function of 25 the incubation time. Fig. 6 shows the release profile as well as the fluorescence spectra of the PSA. The fluorescence intensity of the PSA hardly increased for 2 min of incubation without laser irradiation (off-state). In sharp contrast, a 4-fold increase in the fluorescence intensity of the PSA was observed 30 under laser irradiation with increasing incubation time (onstate). Note, that the release profile of the PSA from the PSAloaded PEGylated GNG was obviously synchronized with the laser-irradiation time, and, consequently, $26 \%$ of the PSA was released from the PSA-loaded PEGylated GNG after 8 35 min of laser irradiation. Additionally, the release of the PSA and the increase in the fluorescence intensity of the PSA were hardly observed for the PSA-loaded PEGylated nanogel (without GNP) even after $8 \mathrm{~min}$ of laser irradiation. These facts strongly suggest that the release of the PSA from the 40 PSA-loaded PEGylated GNGs under laser was due to the deprotonation of the PEAMA gel core through the increase in
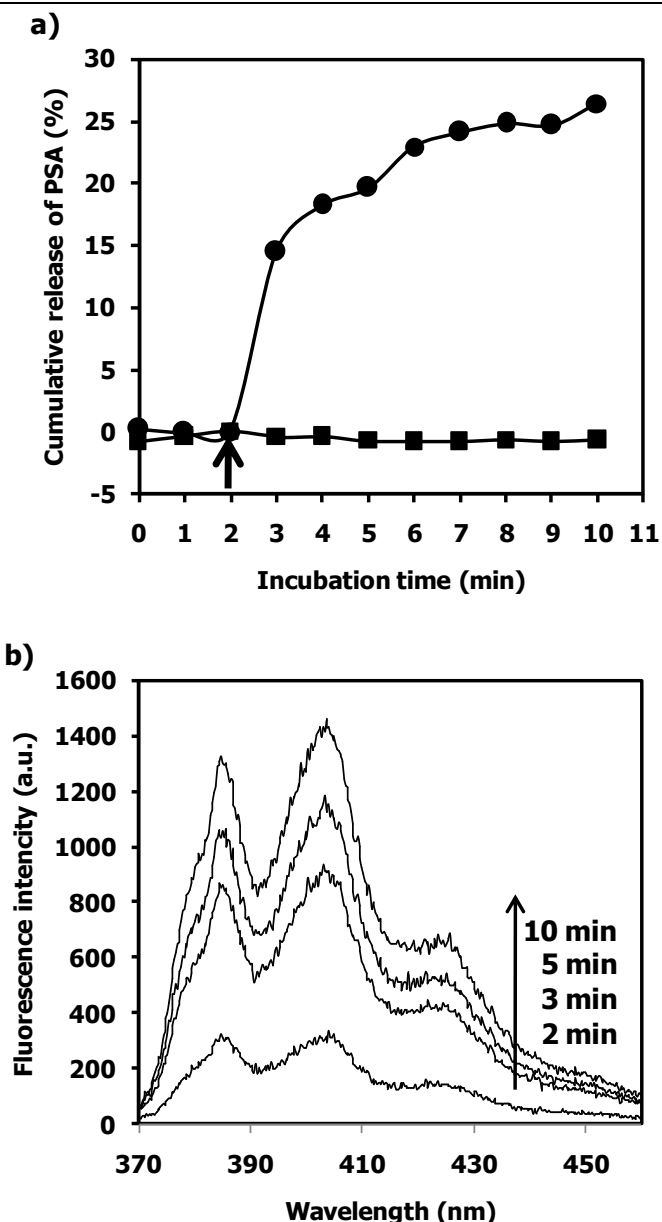

Figure 6. a) Release profile of the PSA from the PSA-loaded PEGylated GNG (circle) and PSA-loaded PEGylated nanogel (square) at $\mathrm{pH} 7.4$ as a function of the incubation time. Irradiation with a $600 \mathrm{~mW} \mathrm{Ar}^{+}$laser $(\lambda=514.5 \mathrm{~nm})$ at a fluence of $39 \mathrm{~W} / \mathrm{cm}^{2}$ was initiated at 2 min (arrow). b) Fluorescence spectra of the PSA for various incubation times $(2,3,5$, and $10 \mathrm{~min})$ in the presence of PSA-loaded PEGylated GNGs.

the temperature of the solution and of the interior of the PEAMA gel core caused by the efficient generation of heat from the GNPs (photothermal effect). Thus, the 45 photothermally-triggered release of the charged dye from PICs composed of PSA and stimuli-responsive PEGylated GNG was performed to achieve the regulation of the fluorescence signals.

In conclusion, the PIC nanoparticles based on smart ${ }_{50}$ PEGylated nanogels containing GNPs and PSA showed the multi-stimuli-triggered release of the PSA in response to $\mathrm{pH}$, temperature, and light, thereby performing the on-off regulation of the fluorescence signal. These findings open the way toward the design of fluorescence sensors as well as 55 controlled drug delivery carriers by the polyion complexation of smart PEGylated nanogels containing GNPs with charged functional molecules.

\section{Acknowledgements.}

This work was partially supported by a Grant-in-Aid for 60 Scientific Research (A) (\#1800033) and the World Premier International Research Center Initiative Program from the 
Ministry of Education, Culture, Sports, Science and Technology of Japan (MEXT).

\section{Notes and references}

${ }^{a}$ Graduate School of Pure and Applied Sciences, University of Tsukuba, 5 1-1-1 Tennoudai, Tsukuba, Ibaraki 305-8573, Japan. Fax: (+81)-29-8535749; Tel: (+81)-29-853-5749; E-mail: nagasaki@nagalabo.jp

${ }^{b}$ Tsukuba Research Center for Interdisciplinary Materials Science (TIMS), University of Tsukuba, 1-1-1 Tennoudai, Tsukuba, Ibaraki 305-8573, Japan.

${ }_{10}{ }^{C}$ Center for Tsukuba Advanced Research Alliance (TARA), University of Tsukuba, 1-1-1 Tennoudai, Tsukuba, Ibaraki 305-8573, Japan

${ }^{d}$ Graduate School of Comprehensive Human Sciences, University of Tsukuba, 1-1-1 Tennoudai, Tsukuba, Ibaraki 305-8573, Japan.

${ }^{e}$ International Center for Materials Nanoarchitectonics Satellite (MANA),

15 National Institute for Materials Science and University of Tsukuba, 1-1-1

Tennoudai, Tsukuba, Ibaraki 305-8573, Japan.

$\dagger$ Electronic Supplementary Information (ESI) available: [details of any supplementary information available should be included here]. See DOI: $10.1039 / \mathrm{b} 000000 \mathrm{x} /$

201 R. Ni, D. Cao, and W. Wang, J. Phys. Chem. B, 2008, 112, 4393.

2 A. Harada, and K. Kataoka, Macromolecules, 1998, 31, 288.

3 K. Kataoka, H. Togawa, A. Harada, K. Yasugi, T. Matsumoto, and S. Katayose, Macromolecules, 1996, 29, 8556.

4 A. V. Kabanov, S. V. Vinogradov, Y. G. Suzdaltseva, and V. Yu. 25 Alakhov, Bioconjugate Chem., 1995, 6, 639.

5 J. Chen, W.-F. Dong, H. Möhwald, and R.Krastev, Chem. Mater., 2008, 20, 1664.

6 R. Ma, B. Wang, X. Liu, Y. An, Y. Li, Z. He, and L.Shi, Langmuir, 2007, 23, 7498.

307 R. Ideta, F. Tasaka, W.-D. Jang, N. Nishiyama, G.-D. Zhang, A. Harada, Y. Yanagi, Y. Tamaki, T. Aida, and K. Kataoka, Nano Lett., $2005, \mathbf{5}, 2426$

8 S. V. Vinogradov, A. D. Zeman, E. V. Batrakova, and A. V. Kabanov, J. Controlled Release, 2005, 107, 143.

359 H. Bysell, and M. Malmsten, Langmuir, 2009, 25, 522.

10 Y. Lee, S. Fukushima, Y. Bae, S. Hiki, T. Ishii, and K. Kataoka, J. Am. Chem. Soc., 2007, 129, 5362.

11 M. Oishi, T, Hayama, Y. Akiyama, S. Takae, A. Harada, Y. Yamasaki, F. Nagatsugi, S. Sasaki, Y. Nagasaki, and K. Kataoka,
12 V. Bulmus, M. Woodward, L. Lin, N. Murthy, P. S. Stayton, and A. S. Hoffman, J. Controlled Release, 2003, 93, 105.

13 Y. Kakizawa, A. Harada, and K. Kataoka, J. Am. Chem. Soc., 1999, 121, 11247.

4514 Y. Kakizawa, and K. Kataoka, Langmuir, 2002, 18, 4539.

15 A. Harada, and K. Kataoka, J. Am. Chem. Soc., 1999, 121, 9241.

16 A. Harada, and K. Kataoka, J. Am. Chem. Soc., 2003, 125, 15306.

17 P. C. Griffiths, C. Alexander, R. Nilmini, S. S. Pennadam, S. M. King, and R. K. Heenan, Biomacromolecules, 2008, 9, 1170.

5018 K. A. Howard, M. Dong, D. Oupicky, H. S. Bisht, C. Buss, F. Besenbacher, and J. Kjems, Small, 2007, 3, 54.

19 Y. Katayama, K. Fujii, E. Ito, S. Sakakihara, T. Sonoda, M. Murata, and M. Maeda, Biomacromolecules, 2002, 3, 905.

20 K. D. Jensen, P. Kope ckova', and J. Kopecek, Bioconjugate Chem., 2002, 13, 975.

21 M.-J. Shieh, C.-L. Peng, P.-J. Lou, C.-H. Chiu, T.-Y. Tsai, C.-Y. Hsu, C. Y. Yeh, and P.-S. Lai, J. Controlled Release, 2008, 129, 200.

22 D. Xu, J. Yu, Y. Liu, H. Sun, J. Xu, K. Sheng, S. Yao, Y. Xu, and H. Lu, Int. J. Nanosci., 2006, 5, 753.

6023 M. Oishi, and Y. Nagasaki, React. Funct. Polym., 2007, 67, 1311.

24 H. Hayashi, M. Iijima, K. Kataoka, Y. Nagasaki, Macromolecules, 2004, 37, 5389

25 M. Oishi, H. Hayashi, M. Iijima, Y. Nagasaki, J. Maer. Chem., 2007, 17, 3720 .

6526 M. Oishi, S. Sumitani, T. K. Bronich, A. V. Kabanov,M. D. Boska, and Y. Nagasaki, Chem. Lett., 2009, 38, 128.

27 M Oishi, S. Sumitani, and Y. Nagasaki, Bioconjugate Chem., 2007, 18, 1379 .

28 A. Tamura, M. Oishi, and Y. Nagasaki, submitted to $70 \quad$ Biomacromolecules in press

29 M. Oishi, A. Tamura, T. Nakamura, and Y. Nagasaki, Adv. Funct. Mater., 2009, 19, 827.

30 M. Oishi, H. Hayashi, T. Uno, T. Ishii, M. Iijima, Y. Nagasaki, Macromol. Chem. Phys., 2007, 208, 1176.

7531 Y. Haba, C. Kojima, A. Harada, T. Ura, H. Horinaka, and K. Kono, Langmuir, 2007, 23, 5243.

32 I. H. El-Sayed, X. Huang, and M. A. El-Sayed, Cancer Lett., 2006, 239, 129.

33 V. P. Zharov,V. Galitovsky, and M. Viegas, Appl. Phys. Lett., 2003, $80 \quad \mathbf{8 3}, 4897$. 